\title{
Strategies to Extend the Network Life Time of MWSN with Self- Organization Clustering
}

\author{
Sun-Young Park \\ Division of Computer Science \\ Keimyung College University \\ sypark@kmcu.ac.kr
}

\begin{abstract}
In wireless sensor networks, the methods for both data collection and data transmission to base station are important factors for sensor node energy consumption. We can transmit the data generated by each node more efficiently using clustering. One of the clustering methods, self-organization clustering, cannot guarantee homogeneous head-node selection. We suggest the adjusted-TTC method, which improves the capacity of the TTC to select a head-node evenly and efficiently in the network field through 2-tier clustering. Adjusted-TTC prioritizes a head-node at the first stage by removing headnodes that are too close to the other head-nodes in the network field. This improves the data transmission energy efficiency among sensor nodes by reducing the number of nonreceiving advertisement messages.
\end{abstract}

Keywords: wireless sensor networks, energy consumption, self-organization, clustering, 2-tier clustering, energy efficiency

\section{Introduction}

Wireless sensor networks consist of a large number of sensor nodes that can recognize the desired information in their environment, analyze and process the data collected, communicate with each other through wireless channels, and thus form a collaborative and self-organizing networks [1]. Energy efficiency and balancing mechanisms are key technologies that warrant close attention in wireless sensor networks [2]. For this reason, many studies have attempted to minimize the energy consumption of sensor nodes [3-7].

Clustering is a technique that can effectively reduce the energy consumption of sensor nodes and has been widely used in WSNs. In clustering protocols, nodes are organized into several clusters according to specific requirements, or metrics, and one of the nodes in a cluster becomes a head-node. Each non-head-node transmits to the head-node instead of sending data to the base-station individually. Compared with flat routing protocols in WSNs, clustering routing protocols have several advantages: such as enhanced scalability, reduced circuit load, increased robustness and reduced energy consumption [8].

As head-nodes are fixed in conventional clustering, sensor nodes acting as heads are expected to consume more energy as compared to other sensor nodes due to their longrange transmission of data to the base station. This results in uneven energy consumption amongst sensor nodes in the network [9].

To solve the problems in conventional clustering, self-organization clustering is suggested in [10]. In self-organizing clustering, we can avoid the problem of rapid energy consumption in specific nodes over that of others by selecting head nodes randomly.

For many applications, such as sea exploration, wildlife protection, and traffic congestion control, mobile sensor nodes need to be deployed in a network. This mobility causes frequent changes in sensor field topology. Mobility therefore becomes a critical issue that must be considered in the design of WSNs with mobile nodes [11]. In MWSNs, it is difficult to adapt self-organization clustering methods 
without some modification. Because the LEACH (Low Energy Adaptive Clustering Hierarchy) protocol using self-organization clustering that assumes a sensor field size of $50 \mathrm{~m} \times 50 \mathrm{~m}$, it is possible to broadcast the Advertisement Message for clustering throughout the sensor field. However, the sensor fields in MWSNs are often larger than those in WSNs. When the sensor field is too large, energy inefficiency may result when head-nodes broadcast the Advertisement Message over the sensor field. Limiting the transmission range of the Advertisement Message is a requirement in this case. Detrimentally, when the transmission range is limited, Advertisement Message non-receiving nodes can be generated due to selforganization clustering. That is because sensors elect themselves to be head-nodes independently in self-organization clustering strategies. The even distribution of headnodes over the entire sensor-network field or even the election of an optimal percentage of head-nodes cannot be guaranteed in this case.

TTC (Two-Tier Clustering) is proposed in [12] to solve the problem of reduced headnodes efficiency in mobile sensor networks. TTC can reduce the number of non-receiving nodes, thus resulting in reduced data loss and increased energy efficiency. Existing selforganization clustering protocols achieve clustering in one-step, whereas TTC consists of a two-step processes. Initially, in order to create clusters in our proposed method, each node decides whether or not to become a head-node according to a pre-determined parameter. Each node that has elected itself a head node broadcasts an Advertisement Message to the other nodes within a radius (R). Non-head-nodes will decide the cluster to which they will belong after they receive a few Advertisement Messages from various head-nodes and compare their signal strengths. After each node has selected its cluster, it must inform the head-node that it will be a member of that cluster. That is the first step in our clustering method. After a predefined waiting time, the nodes that have not received any Advertisement Message decide once again whether or not to become a head-node. The nodes that have become head-nodes reconfigure their clusters as they did in the first step.

TTC can reduce the number of nodes which do not belong to clusters compared with existing self-organization clustering protocols for any arbitrary (R). However, this process still generates head-nodes that are distributed too closely.

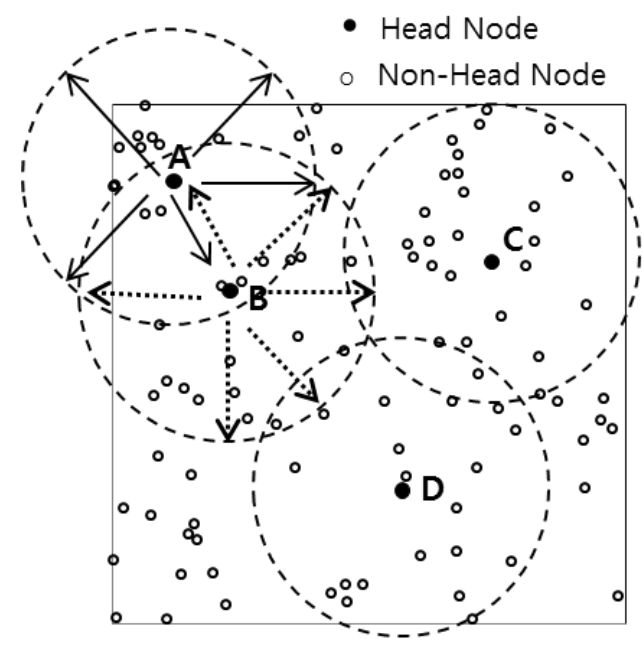

Figure 1. Process of Configuring Clusters in an Adjusted-TTC (Step 1) 
In this paper, we suggest adjusted-TTC to improve the even distribution of head-nodes in a network field as much as possible. Adjusted-TTC removes multiple head-nodes that are selected in the same cluster, resulting in the even distribution of head-nodes in the sensor field as well as reducing the number of non-receiving nodes. Adjusted-TTC selects head-nodes through two steps. After we generate the head-nodes at the first step, we remove head-nodes that are too close to existing ones. At the second step, we generate the pre-determined number of head-nodes and additionally create the same number of headnodes as removed at the first step.

Figure 1 show an example where two adjacent head-nodes, A and B, are so close that they receive Advertisement Messages from each other. When node A has more energy than node $\mathrm{B}$, it remains as a head-node while node $\mathrm{B}$ becomes a normal sensor node. In the second step of clustering, all the other nodes except head-nodes A, C, D, can participate in further head-node selection at the second step of clustering.

In adjusted-TTC, Advertisement Messages also report the remaining energy in each head- node and this message requires extra time for the transmission of a relinquishment message to nodes that are disqualified from becoming head-nodes. However, this extra transmission time and resultant energy usage is more than compensated for through a reduction in the number of nodes that are not included in clusters, shortened data transmission distances, and a significant increase in overall sensor field efficiency.

The rest of this paper consists of as follows: In Section 2, we explain the operational process of TTC strategy. In Section 3, we describe the adjusted-TTC. In Section 4, we analyze the additional number of the head node and calculate threshold for step 2 clustering. In Section 5, we present the results of our simulation in order to evaluate our adjusted-TTC and other protocols. We conclude our paper in Section 6.

\section{Previous Work}

In self-organization clustering, if a non-head-node does not receive any Advertisement Message, it cannot belong to any cluster and therefore cannot successfully transfer its sensing data through a head-node to the base station.

In existing self-organization clustering protocols, clustering is performed at the set-up phase: consisting of head-node election, transmission of an Advertisement Message and the join-cluster decision of non-head-nodes. The basic idea of TTC proposed in [12] is to perform the set-up phase twice to reduce the number of non-receiving nodes that are excluded from clusters.

Assume $N$ sensors are distributed randomly in a network field. In TTC, let $p^{\prime}$ as the rate of head nodes in the first step and $p^{\prime \prime}$ as the rate of head-nodes in the second step.

The rate, $\mathrm{p}$ to be a head-node in the number among $\mathrm{N}$ sensor nodes will be:

$$
p=p^{\prime}+p^{\prime \prime}
$$

For the first step in clustering, each node decides whether or not to become a headnode independently. Each node generates a random number between 0 and 1 and compares that number to the threshold $\theta^{\prime}$ calculated by a predetermined rate of headnodes and the number of transmission rounds. If the former is smaller than the latter, the node becomes a head- node.

$$
\theta^{\prime}=\frac{p^{\prime}}{1-p^{\prime}\left(r \bmod 1 / p^{\prime}\right)}
$$

The nodes that have become head-nodes broadcast an Advertisement Message to the rest of the nodes within a radius of $(R)$. Each non-head-node decides the cluster to which it will belong for each round. This decision is based on the signal strength of the received Advertisement Message. After each node has determined its headnode, it sends that head-node a response message. The nodes which have not received any Advertisement Message in the first step perform the second step of clustering. 
$N^{\prime}$ denotes a set of nodes which have not been included in any clusters at the first step. $\theta^{\prime \prime}$ is the threshold that the nodes in set $N^{\prime}$ use to decide whether or not they will become head-nodes at the second step. All nodes in set $N^{\prime}$ can independently calculate the threshold $\theta^{\prime \prime}$.

To generate $p^{\prime \prime} \cdot N$ head-nodes among the nodes in set $N^{\prime}$, the threshold $\theta^{\prime \prime}$ is :

$$
\theta^{\prime \prime}=\frac{p^{\prime \prime}}{\left(1-\theta^{\prime}\right)^{\lambda}}
$$

$\left(1-\theta^{\prime}\right)^{\lambda}$ is the rate of non-receiving nodes after the first step in clustering. Each non-receiving node from the first step generates a random number and compares it with the threshold $\theta^{\prime \prime}$ for the second step. If the generated random value is less than $\theta^{\prime \prime}$, then the node becomes a head-node. Elected head-nodes broadcast an Advertisement Message to the rest of the nodes within a radius of $(R)$. In the second step, each non-head-node sends a response message to the head-node after it decides which cluster it will belong to by repeating the same process as in the first step. The nodes that have received Advertisement Messages from several head-nodes respond to the head-node that has broadcast the strongest Advertisement Message signal.

\section{Adjusted-TTC}

TTC could reduce the numbers of non-receiving nodes effectively through a two-tier head-node decision process. However, either LEACH-Mobile or TTC sometimes cannot avoid producing more than two head-nodes in the same cluster. Adjusted-TTC could reduce this problem by eliminating multiple head-nodes selected in the same cluster field at the first step and then create the same number of head-nodes at the second step to produce head-nodes evenly throughout the network field. In this chapter, we explain how adjusted-TTC works.

This is the head-node selection process in adjusted-TTC. All head-nodes that are selected at the first step send Advertisement Messages to all the other nodes in the same cluster. At this time, each head-node reports its remaining energy reserve. All head-nodes that received Advertisement Messages from other head-nodes compare their remaining energy with the sender's remaining energy. If its own remaining energy is larger than the sender's is, then it remains a head-node. If its own remaining energy is smaller than the sender's is, then this head-node sends a relinquishment message to all the nodes to which it sent an Advertisement Message.

In Figure 2, nodes $\mathrm{N}_{3}, \mathrm{~N}_{4}, \mathrm{~N}_{7}$ are selected as head-nodes at the first step. These headnodes send Advertisement Messages to the nodes that are within transmission range. All the other nodes except node $\mathrm{N}_{7}$ receive an Advertisement Message. Node $\mathrm{N}_{3}$ receives an Advertisement Message from node $\mathrm{N}_{4}$ and node $\mathrm{N}_{4}$ receives an Advertisement Message from node $\mathrm{N}_{3}$. Because node $\mathrm{N}_{3}$ and $\mathrm{N}_{4}$ are both head-nodes, they compare their remaining energy with each other. Therefore, if node $\mathrm{N}_{3}$ has less remaining energy than node $\mathrm{N}_{4}$, that node $\mathrm{N}_{3}$ sends a relinquishment message to the other nodes, to which it has previously sent an Advertisement Message, disqualifying itself as a head-node. In $\mathrm{T}_{\mathrm{c} \text {-select }}$, as all other nodes except node $\mathrm{N}_{1}$ (node $\mathrm{N}_{3}$ was discarded as a head-node) and all other head-nodes send response messages to each head-node, to which it had previously sent an Advertisement Message, reporting which head-node they belong to. Node $\mathrm{N}_{1}$, which does not yet belong to any cluster, participates in the head-node selection process at the second step of clustering. 


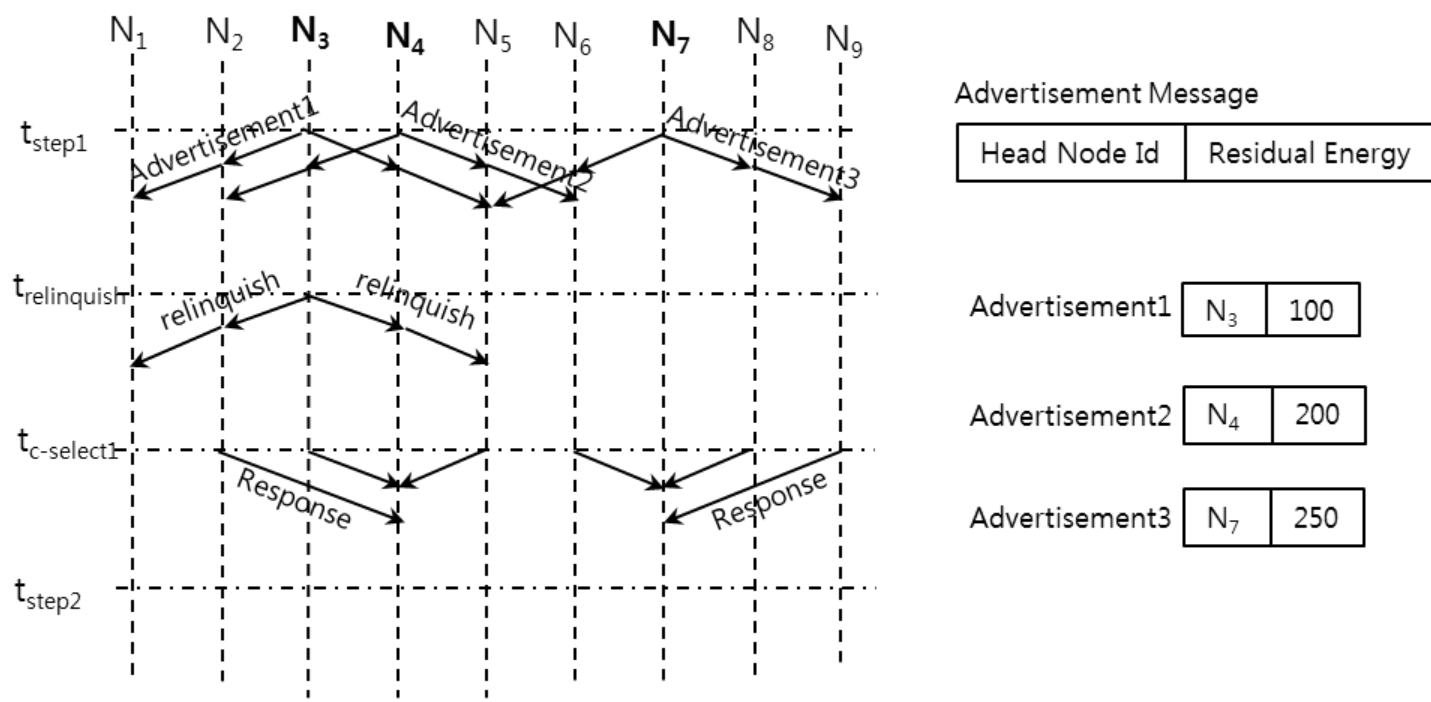

Figure 2. The Signal Sending Process for Making Clustering

In the second step of clustering, we generate a pre-decided number of head-nodes plus the number of head-nodes discarded at the first step. In the second step of clustering, all selected head-nodes form their own clusters. This clustering process is same as TTC's.

It is important to get rid of adjacent head-nodes at the first step because the number of head- nodes generated by the first step is greater than the number from the second step.

\section{Calculating Threshold for Head-Node Selection in Step 2}

Our scheme eliminates head-nodes if there are two or more adjacent at the first step and additionally generates an equivalent number of head-nodes at the second step. In this chapter, we analyze the number of head-nodes that are removed at the first step and calculate a threshold for creating head-nodes that should be generated at the second step.

When $\mathrm{R}$ is the radius of a cluster, let $\lambda_{R}$ be the average number of nodes in the cluster, and let $\theta$ be the probability to become a head-node, then the probability of the number of head-nodes in the cluster is $\mathrm{X}$ and this is a binominal probability distribution expressed as[13]:

$$
X \sim \mathrm{B}\left(\lambda_{R}, \theta\right)
$$

$P(X=x)$ is the probability that $X=x$.

$$
P(X=x)=\left(\begin{array}{c}
\lambda_{R} \\
x
\end{array}\right) \theta^{x}(1-\theta)^{\lambda_{R}-x}
$$

If there are more than two head-nodes generated in a cluster, all the other head-nodes in the cluster have to be disqualified while one remains. The probability of generating more than two head-nodes is:

$$
\mathrm{P}(\mathrm{X} \geq 2)=\sum_{x=2}^{\lambda_{R}} P(X=x)
$$

When $\mathrm{R}$ is the radius of a cluster, we calculate the number of head-nodes that are disqualified in a cluster as:

$$
H_{R}=\sum_{x=2}^{\lambda_{R}}(P(X=x) \times(x-1))
$$

We also calculate the total number $F H_{R}$ of disqualified head-nodes by multiplying number of disqualified head-nodes from expression (7) and the number of clusters.

$$
F H_{R}=H_{R} \cdot N / \lambda_{R}
$$

We calculate the rate $\alpha_{R}$ for the number of head-nodes to be additionally generated in the second step of clustering.

$$
\alpha_{R}=F H_{R} \cdot N / \lambda_{R}
$$


We can calculate the threshold $\left(\theta^{\prime \prime}\right)$ for the generation of head-nodes in the second step by using the rate of head-nodes for the second step $\left(p^{\prime \prime}\right)$ and the rate of additional head-nodes $\left(\alpha_{R}\right)$.

$$
\theta^{\prime \prime}=\frac{p^{\prime \prime}+\alpha_{R}}{\left(1-\theta^{\prime}\right)^{\lambda_{R}}}
$$

$\left(1-\theta^{\prime}\right)^{\lambda_{R}}$ is the rate of non-receiving nodes after the first step of clustering. Each non-receiving node from the first step generates a random number and compares it with the threshold $\theta^{\prime \prime}$ for the second step.

\section{Performance Evaluation}

To evaluate the performance of our adjusted-TTC, we simulate LEACH-mobile and TTC together.

We assume 1000 nodes are scattered randomly over a $1000 \mathrm{~m}$ X $1000 \mathrm{~m}$ sensor field. The total percentage of head-nodes is $5 \%$.

Table 1. Simulation Parameter

\begin{tabular}{ll}
\hline Parameter & Value \\
\hline Number of sensor nodes(N) & 1000 \\
Network size(F) & $1000 \times 1000$ \\
Node deployment mode & Random distribution \\
Percentage of cluster heads in step $1\left(p^{\prime}\right)$ & $4 \%$ \\
Percentage of cluster heads in step $2\left(p^{\prime \prime}\right)$ & $1 \%$ \\
\hline
\end{tabular}

In LEACH-Mobile, each node calculates a threshold $\theta$ using formula (11) and generates a random number for the threshold to decide for itself to become a head-node or not [14].

$$
\theta=\frac{p}{1-p(r \bmod 1 / p)}
$$

Then, each self-elected head-node broadcasts an Advertisement Message to the sensor nodes within its transmission radius. At this time, the nodes that cannot receive the Advertisement Message will become non-receiving nodes.

In TTC and adjusted-TTC, we suppose the percentage of head-nodes is $4 \%$ in the first step of head-node selection and $1 \%$ in the second step in order to generate the same percentage of head-nodes as in LEACH-Mobile. In adjusted-TTC, the number of head-nodes additionally generated in the second step is the same as the number of head-nodes eliminated in the first step.

Figure 3 shows the average number of non-receiving nodes that cannot receive an Advertisement Message that were generated through simulations using each clustering strategy.

From the results of this simulation, adjusted-TTC reduces the number of non-receiving nodes by more than $29 \%$ compared with LEACH-Mobile and achieves a $19 \%$ reduction when compared with TTC for a transmission radius of $80 \mathrm{~m}$. As the transmission radius increases, the number of non-receiving nodes is reduced. In adjusted-TTC, when the transmission radius is $140 \mathrm{~m}$, a more than $85 \%$ reduction in the number of non-receiving nodes is achieved compared with LEACH-mobile and a $39 \%$ reduction is achieved compared with TTC. 


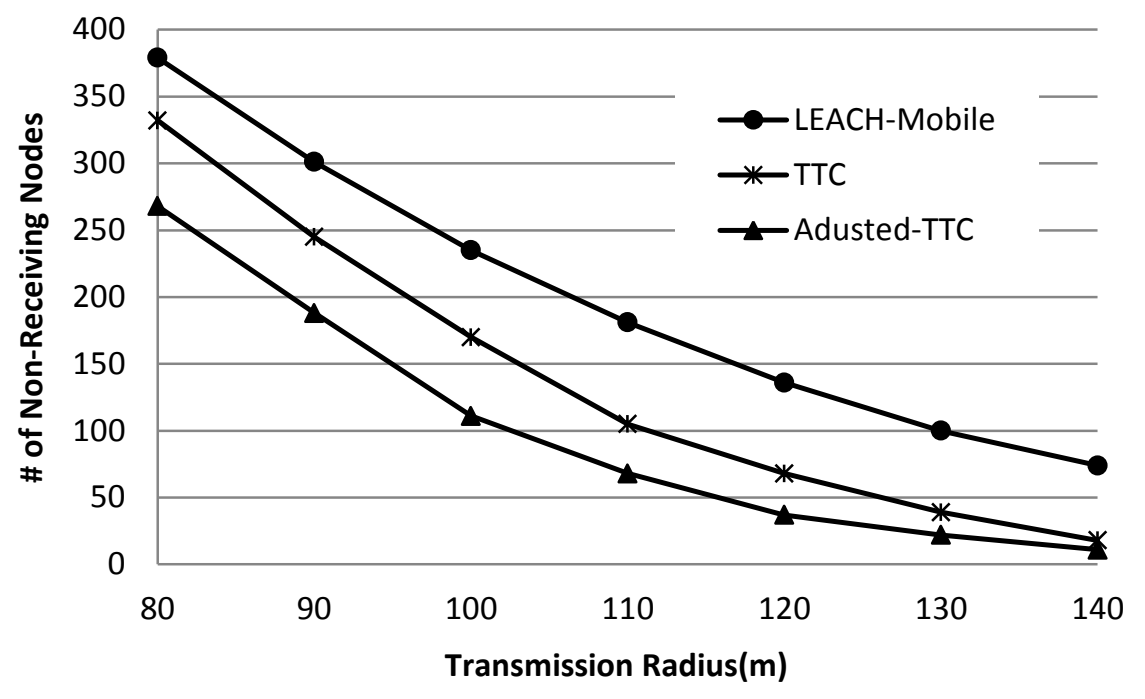

Figure 3. Comparison of the Number of Non-Receiving Nodes

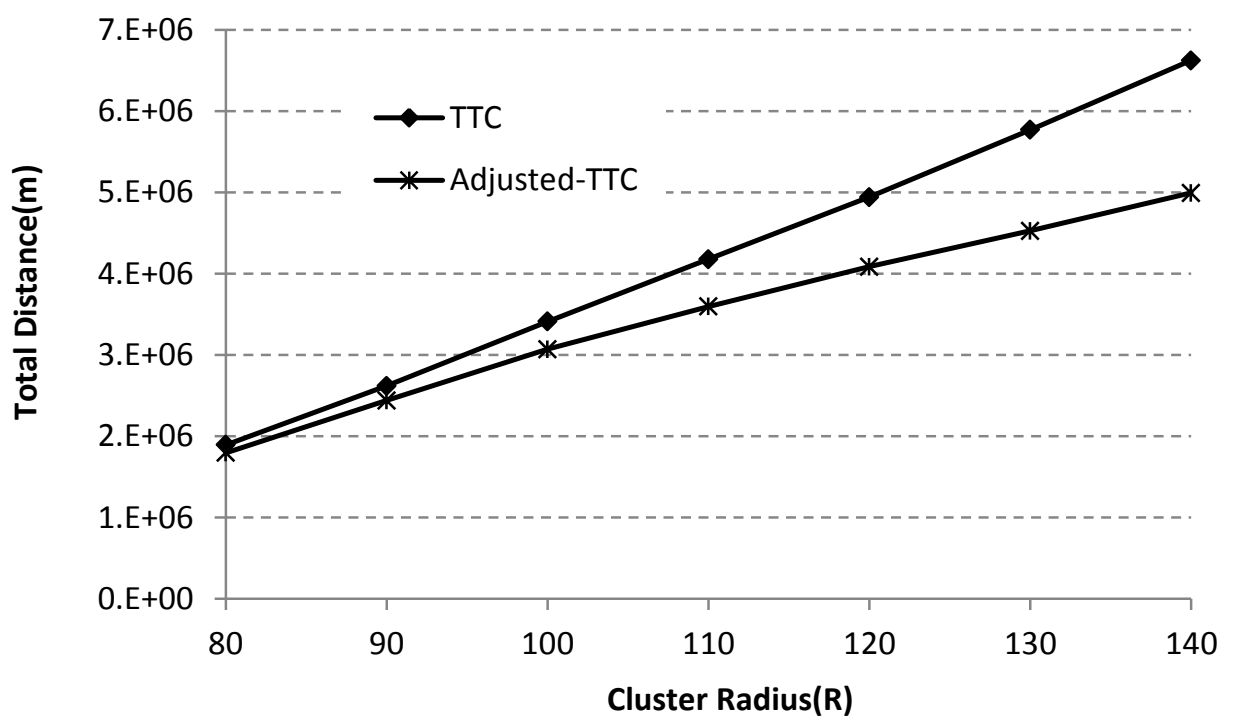

Figure 4. Comparison of Distances between Node and Head-Node in TTC and Adjusted-TTC

Figure 4 shows the sum of the distances between head-nodes and sensor nodes as the cluster size varies in each method. As the cluster size grows larger, the total distance between heads-nodes and sensor nodes in adjusted-TTC becomes smaller than in TTC. Especially when the transmission radius is large, if head-nodes are not distributed homogeneously then the sum of the head-nodes and sensor nodes becomes too large.

Figure 5 shows the state of dispersion after clustering simulation using adjusted-TTC when field radius $\mathrm{R}=90$. Figure 6 and Figure 7 show the states of dispersion after clustering simulation using TTC and LEACH-Mobile respectively. This shows that clustering using adjusted-TTC is more homogeneous than either TTC or LEACH-Mobile. 


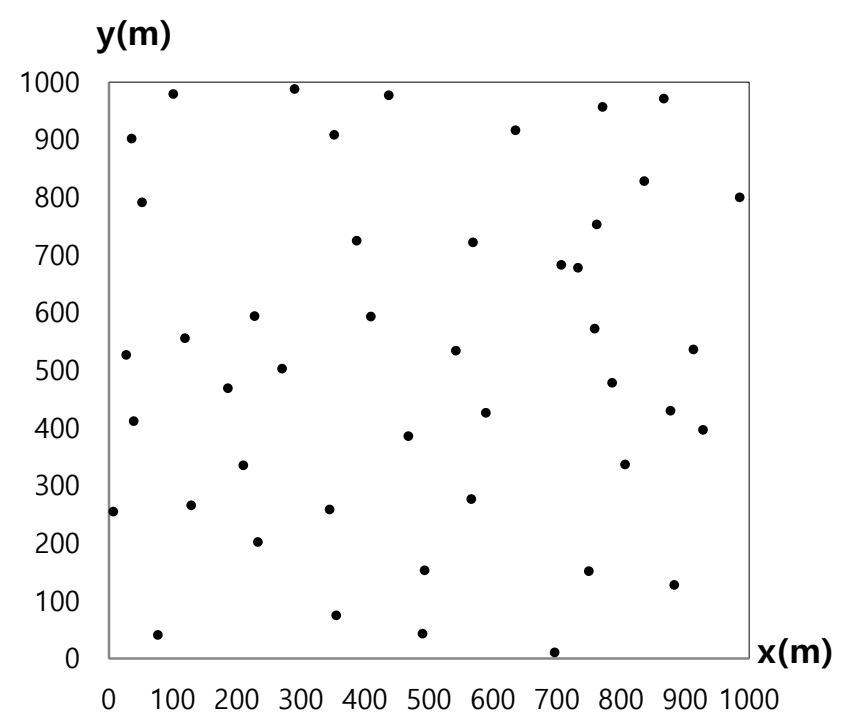

Figure 5. Distribution of Head-Nodes in Adjusted-TTC (R=90)

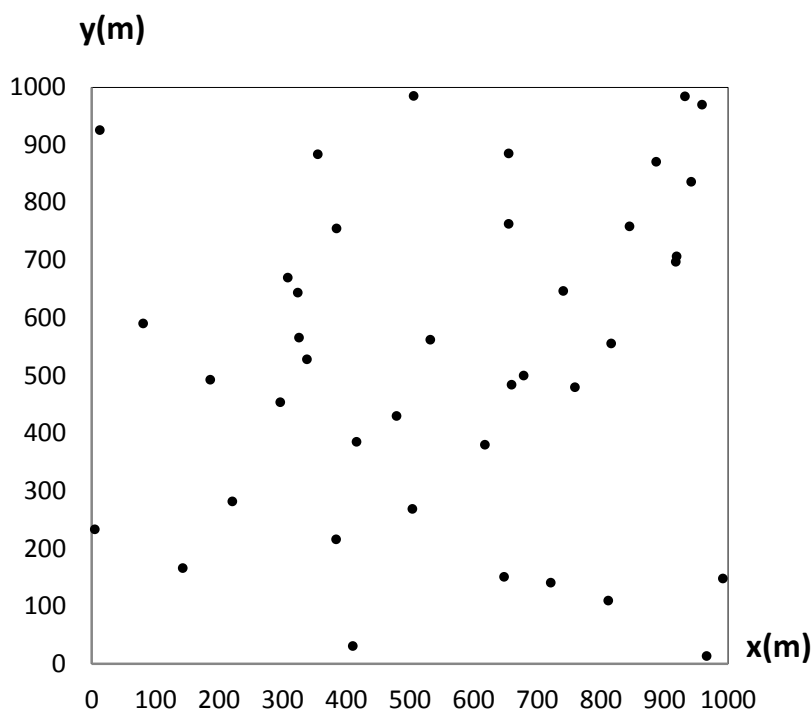

Figure 6. Distribution of Head-Nodes in TTC $(R=90)$ 


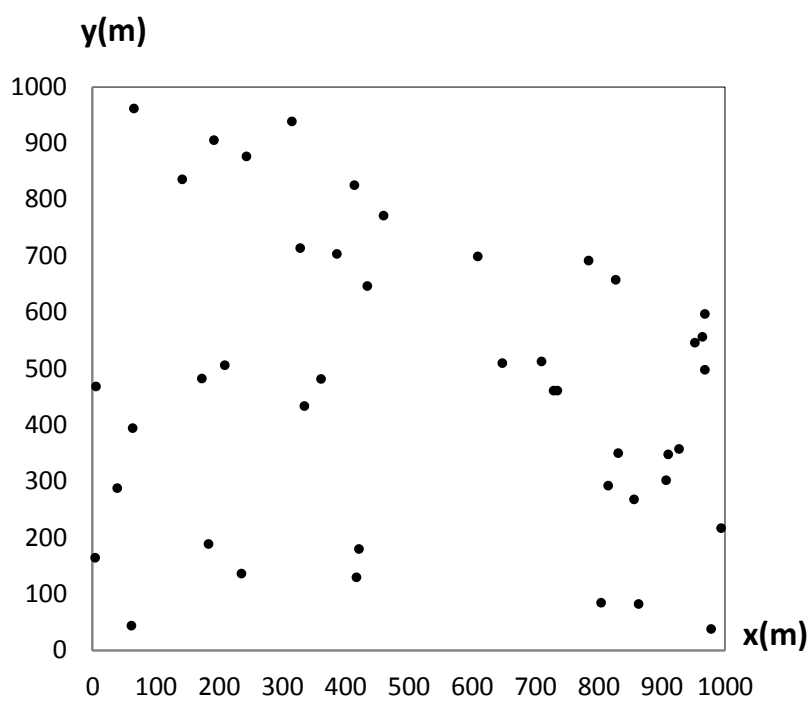

Figure 7. Distribution of Head-Nodes in LEACH-Mobile ( $R=90)$

\section{Conclusion}

Clustering is an energy efficient method in collecting and transmitting data from sensor networks. TTC ameliorates the problems of self-organizational clustering with its efficient clustering.

In this paper, we suggest adjusted-TTC as a preferred method to further improve the efficiency of TTC. Adjusted-TTC improves the index of dispersion by disqualifying multiple adjacent head-nodes in the same clustering field at the first step of clustering. This method results in a shortened distance between each head-node and the surrounding sensor nodes resulting in increased overall sensor-field energy efficiency.

To verify the improvements realized in adjusted-TTC, we simulate LEACH mobile, TTC and adjusted-TTC. As a result, the number of non-receiving nodes is reduced in adjusted-TTC compared with both LEACH-Mobile and TTC. In addition, we calculate the total distance between head-nodes and sensor nodes for each method. We find the total distance in adjusted-TTC is significantly shorter than the distances for the other two methods.

\section{References}

[1]. Z. Wang, X. Zhao, X. Qian and X. Cheng, "The optimal transmission range for strip-based wireless sensor networks", Applied Mechanics and Materials, vol. 121-126, (2012), pp. 4213-4217.

[2]. X.-H. Deng, C. Xu and Y. Liu, "Energy Balanced Scheme Based on Variable Cell Transmission Range for Wireless Sensor Networks", 5th International Conference on Wireless Communications, Networking and Mobile Computing, WiCOM, (2009).

[3]. H. Kim, "An Efficient Clustering Scheme for Data Aggregation Considering Mobility in Mobile Wireless Sensor Networks", International Journal of Control and Automation, vol. 6, no. 1, (2013) February, pp. 221-234.

[4]. E. Lee, S. Park, F. Yu and S.-H. Kim, "Data Gathering Mechanism with Local Sink in Geographic Routing for Wireless Sensor Networks", IEEE Trans. on Consumer Electronics, vol. 56, (2010), pp. 1433-1441.

[5]. S.-H. Hong and B.-K. Kim, "An Efficient Data Gathering Routing Protocol in Sensor Networks Using the Integrated Gateway Node”, IEEE Trans. on Consumer Electronics, vol. 56, (2010), pp. 627-632.

[6]. M. Lee, "Enhanced Shortest-Path Technique to Reduce Transmission Power in Wireless Sensor Networks", International Journal of Control and Automation, vol. 6, no. 3, (2013) June, pp. 395-404.

[7]. M. Lehsainil, H. Guyennet and M. Feham, "Cluster-based Energy efficient Scheme for Mobile Wireless Sensor Networks, Wireless Sensor and Actor Networks II”, The International Federation for Information 
Processing, vol. 264, (2008), pp. 13-24.

[8]. X. Lin, "A Survey on Clustering Routing Protocols in Wireless Sensor Networks, Sensors, (2012), pp. 11113-11153.

[9]. B. P. Deosarkar, N. S. Yadav and R. P. Yadav, "Cluster head Selection in Clustering Algorithms for Wireless Sensor Networks", A Survey, Proceedings of the 2008 International Conference on Computing and Communication and Networking, (2008).

[10].W. R. Heinzelman, A. Chandrakasan and H. Balakrishnan, "Energy-Efficient Communication Protocol for Wireless Microsensor Networks", Proceedings of the 33rd Hawaii International Conference on System Sciences, (2000), pp. 1-10.

[11].S. Deng, J. Li and L. Shen, "Mobile-based clustering protocol for wireless sensor networks with mobile nodes", IET Wireless Sensor System, vol. 1, Issue 1, (2011), pp. 39-47.

[12].S.-Y. Park, "An efficient clustering scheme for cluster-based protocols in mobile wireless sensor networks", International Journal of Control and Automation, vol. 7, no. 9, (2014), pp. 375-388.

[13].Y.-L. Jeon, "Utilization of Statistical Theory for Science and Engineering”, GS Intervision, (2013), pp. 94-100.

[14].D. S. Kim and Y. J. Chung, "Self-organization routing protocol supporting mobile nodes for wireless sensor networks", Proceedings First Int. MultiSymp. On Computer and Computational Sciences, Hangzhou, China, (2006) June, pp. 622-626.

\section{Author}

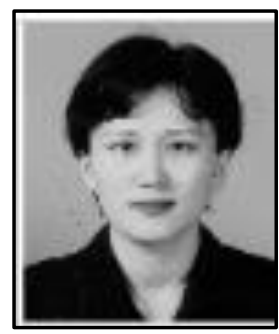

Sun-Young Park, Professor Park received her B.S., M.E. and Ph.D. degrees in computer engineering from Kyung-Pook National University, Republic of Korea, in 1990, 1993 and 1998 respectively. She has been a professor in the Department of Computer Science at Kei-Myung College University, Republic of Korea, since 1998. Her research interests include various aspects of Wireless Sensor Networks, Wireless Mobile Communication and Wireless Communication Protocols. 